\title{
ANÁLISE DO CLIMA ORGANIZACIONAL: UM ESTUDO MULTICASO EM EMPRESAS DO ESTADO DO PARANÁ.
}

\author{
Jean Francisco Bernardino ${ }^{1}$ \\ Helena de Fátima Nunes Silva ${ }^{2}$ \\ Fernanda Cristina Barbosa Pereira Queiroz ${ }^{3}$
}

\begin{abstract}
RESUMO: A compreensão do clima organizacional pode auxiliar na gestão das estratégias que orientam as intervenções e mudanças necessárias ao crescimento e desenvolvimento das organizações. Este artigo tem como objetivo analisar as informações a respeito do clima organizacional de três empresas paranaenses, de diferentes ramos de atividades, denominadas A, B e C. A Empresa A fornece serviços e tecnologias para uma instituição bancária, a Empresa B realiza serviços essenciais em um município, como limpeza pública e a Empresa $\mathrm{C}$ atua na área de transporte de grãos. Para a coleta de dados utilizou-se um questionário estruturado, contendo 22 questões objetivas, abrangendo as seguintes variáveis: avaliação da supervisão, avaliação do salário, avaliação do desenvolvimento profissional, avaliação do feedback e avaliação do trabalho em si (nível de estresse dos funcionários). Os resultados encontrados indicam que as Empresas A e B estão dentro do padrão de clima organizacional considerado regular e a Empresa $\mathrm{C}$ pode ser enquadrada como um clima ruim. Conclui-se que há similaridade entre as empresas em alguns quesitos, que estão relacionados ao negócio, estrutura e estratégia organizacional.
\end{abstract}

Palavras-chave: Clima Organizacional. Comportamento Organizacional. Gestão de Pessoas.

ABSTRACT: The understanding of the organizational climate can help in the management of strategies that guide interventions and changes needed to the growth and development of organizations. This article aims to analyze the information about the organizational climate of three organizations located in the state of Paraná (Brazil), from different branches of activities, called A, B and $\mathrm{C}$. The organization A provides services and technologies to a bank, the company B performs essential services in a city, such as public cleaning and the $\mathrm{C}$ Company operates in grain transportation. A structured questionnaire containing 22 objective questions used for data collection with the following variables: evaluation of supervision, salary, professional development, feedback and the work itself (stress level among employees). The results found in the article indicate that Companies A and B can be

\footnotetext{
${ }^{1}$ Mestrando, Universidade Federal do Paraná, e-mail: jean.f.bernardino@gmail.comfer

${ }^{2}$ Professora Doutora, PPCGI, Universidade Federal do Paraná - UFPR. helenanunes@ufpr.br

3 Professora Doutora, PPGEP, Universidade Federal do Rio Grande do Norte - UFRN. fernandacbpereira@gmail.com
}

ReLAInEP - Revista Latino-America de Inovação e Engenharia de Produção, Curitiba, PR, Brasil, v. 3, n. 4, p. 131-151, 2015. 


\section{ReLAInEP}

considered as a regular organizational climate and the Company $\mathrm{C}$ can be framed as a bad organizational climate. It concluded that there is similarity between the companies in some questions, which are related to the business, organizational structure and strategy.

Keywords: Organizational Climate. Organizational Behavior. Human Management.

RESUMEN: La comprensión del clima organizacional puede auxiliar en la gestión de las estrategias que orientan las intervenciones y cambios necesarios para el crecimiento y desarrollo de las organizaciones. Este artículo tiene como objetivo analizar las informaciones al respecto del clima organizacional de tres empresas paranaenses, de distintos ramos de actividades denominadas como A, B y C. La empresa A provee de servicios y tecnologías para una institución bancaria, la empresa $\mathrm{B}$ realiza servicios esenciales en un municipio, como limpieza pública, y la empresa $\mathrm{C}$ actúa en el transporte de granos. Para la colecta de datos fue utilizado un cuestionario estructurado, el cual comprendió de 22 preguntas objetivas, abarcando las siguientes variables: evaluación de la supervisión, evaluación del salario, evaluación del desarrollo profesional, evaluación de la retroalimentación y evaluación del trabajo, en esta última, se refiere al nivel de estrés de los funcionarios. Los resultados encontrados indican que las empresas A y B están dentro de un mal clima. Concluyéndose que hay similitudes entre las empresas en algunas cuestiones relacionadas al tipo negocio, estructura y estrategia organizacional.

Descriptores: Clima organizacional. Comportamiento Organizacional. Gestión de Personas.

\section{INTRODUÇÃO}

Pretendeu-se neste artigo identificar a percepção dos colaboradores das empresas selecionadas em relação às variáveis que influenciam o clima organizacional. Nesta perspectiva, esta pesquisa auxilia empresas de porte ou ramo de atividade variados a tratarem de problemas situacionais internos, utilizando-os como fonte de dados para avaliação de todo o processo. Sendo assim trata-se de uma importante ferramenta gerencial que pode ser utilizada a fim de propiciar estratégias de aprendizagem, solucionar problemas de clima organizacional e a evitá-los.

O clima organizacional não é algo simples de ser mensurado e analisado nas empresas, devido à multidimensionalidade das variáveis que interferem nas relações dos colaboradores com o ambiente de trabalho. Uma definição precisa e ampla para o clima organizacional não existe, trata-se de um conceito que é influenciado pelas percepções dos indivíduos de um determinado nível hierárquico dentro das organizações (HELLRIEGEL E SLOCUM, 1974).

ReLAInEP - Revista Latino-America de Inovação e Engenharia de Produção, Curitiba, PR, Brasil, v. 3, n. 4, p. 131-151, 2015. 


\section{ReLAInEP}

De acordo com Kolb, Rubin e Mcintyre (1978) é importante que o gestor compreenda as variáveis que influenciam o clima organizacional, porque é por meio desse conhecimento que ele pode manejar a motivação de seus empregados. A eficiência da organização pode ser aumentada por meio da criação de um clima organizacional que satisfaça as necessidades de seus membros e, ao mesmo tempo, canalize esse comportamento motivado em direção aos objetivos organizacionais.

Corroborando esta visão, Araújo e Garcia (2009) colocam que o gestor de pessoas deve ser hábil no sentido de identificar eventuais ruídos nos relacionamentos entre as pessoas, visando o melhor clima possível, assegurando um desenvolvimento regular dos trabalhos na organização.

Portanto, o clima organizacional está ligado, diretamente, à maneira como o colaborador percebe a organização, sendo influenciado pela cultura, normas, usos e costumes; como ele interpreta tudo isso e como ele reage, positiva ou negativamente, a essa interpretação. Para Luz (2007, p. 9), “clima organizacional retrata o grau de satisfação das pessoas no ambiente de trabalho".

A organização é um universo com posturas pessoais e profissionais diferenciadas, difícil encontrar uma só empresa em que todas as pessoas que dela participem tenham conceitos e valores uniformes. Alguns terão uma percepção positiva, e a considerarão uma situação agradável ou motivadora, porque de alguma maneira ela atende as suas aspirações e desejos. Outros, frente à mesma situação, terão uma percepção negativa porque, de maneira contrária, ela não atende suas motivações.

Dentro de uma mesma organização, diferentes climas organizacionais são criados, não é possível se falar da existência de apenas um clima organizacional único e uniforme; o clima irá variar de acordo com a situação psicológica e o estágio motivacional vigente, além de considerar cada colaborador e a maneira como este interpreta o contexto da empresa, por meio das informações recebidas ou percebidas no ambiente interno.

Conforme Araújo e Garcia (2009) o processo de comunicação se desenrola nas organizações, a existência de ruídos, termo técnico para a distorção do processo, ocorre com frequência. Some-se a isso a instabilidade e desconfiança nos processos. Ruídos não seriam apenas problemas nos equipamentos, mas também entendimento de mensagens, permitindo

ReLAInEP - Revista Latino-America de Inovação e Engenharia de Produção, Curitiba, PR, Brasil, v. 3, n. 4, p. 131-151, 2015. 


\section{ReLAInEP}

dupla interpretação. "O estudo do clima representa um instrumento de avaliação do modelo de gestão e da política de desenvolvimento organizacional" (TACHIZAWA; FERREIRA; FORTUNA, 2004, p. 243).

Este artigo está organizado nesta primeira seção introdutória, logo após é apresentado o referencial teórico com ênfase nas variáveis do clima organizacional. Em seguida, a metodologia para a elaboração do mesmo e os resultados encontrados com a pesquisa exploratória. Por fim, são apresentadas as considerações finais a respeito dos casos analisados e as referências utilizadas.

\section{REFERENCIAL TEÓRICO}

Nesta seção são apresentadas as bases teóricas que contribuíram para construção do instrumento de coleta e para a análise dos dados, destacando as variáveis que afetam a visão dos colaboradores sobre o clima organizacional.

\subsection{Clima Organizacional}

Existem diversas definições sobre clima e cultura organizacionais e algumas vezes algumas confusões nas análises destes dois fenômenos. Denison (1996) revisou a literatura e concluiu que devido ao grande número de variáveis usadas para medir o clima organizacional, e a inexistência de consenso sobre a definição das mesmas, muitos autores confundem clima com cultura organizacional. Contudo, esses dois conceitos se originaram de disciplinas diferentes e tratam de aspectos complementares, porém distintos no ambiente organizacional. Neste artigo, a análise está centrada nas variáveis que afetam o clima organizacional.

Conforme Menezes e Gomes (2010), as premissas dos estudos sobre clima organizacional podem ser encontradas no experimento conduzido por Elton Mayo em 1927, conhecido como Experimento de Hawthorne. Naquela ocasião, Mayo constatou que modificações nas configurações física e social do ambiente de trabalho podem provocar mudanças emocionais e comportamentais significativas nos trabalhadores, como fadiga, acidentes de trabalho, turnover e redução no desempenho e produtividade (ASHKANASY, WILDEROM E PETERSON, 2000 Apud MENEZES E GOMES, 2010).

ReLAInEP - Revista Latino-America de Inovação e Engenharia de Produção, Curitiba, PR, Brasil, v. 3, n. 4, p. 131-151, 2015. 


\section{ReLAInEP}

Na década seguinte, o termo clima foi cunhado por Lewin, Lippitt e White (1939, apud BEGNAMI e ZORZO 2013), advindo do vocabulário da psicologia social utilizando conceitos similares como clima social e atmosfera social para descrever atitudes, sentimentos e processos sociais. Para Lewin (1951), todo comportamento (ação, pensamento, desejo) busca valorização e realização, que demanda mudança de algum estado de um campo numa determinada unidade de tempo.

O conceito de clima organizacional tem sido fortemente definido desde a década de 60. Os primeiros pesquisadores a estudarem o constructo foram Forehand e Guilmer (1964, p. 362) que o definiram como: "o conjunto de características que descreve uma organização e que: a) a distinguem de outras, b) são relativamente duradouras ao longo do tempo; e c) influenciam o comportamento das pessoas na organização.

Este conceito vem mudando ao longo do tempo, sendo que a percepção e motivação dos funcionários passaram a ser palavras chaves. De acordo com Abbey e Dickson (1983) o clima organizacional pode ser entendido como uma medida de qualidade de um ambiente interno de uma organização, resultante do comportamento e conduta dos seus membros, servindo como uma base para interpretar a situação e agir, e também, como uma fonte de pressão direcionando as atividades.

Tachizawa, Ferreira e Fortuna (2004) referem-se ao clima organizacional como o ambiente interno das organizações que pode ser percebido ou experimentado, age sobre o comportamento dos trabalhadores e interfere no seu grau de motivação e satisfação.

Para Lemos e Martins (2007, p. 6) o clima organizacional representa o somatório das percepções individuais geradas do ambiente organizacional. Trata-se, portanto, de um fenômeno coletivo contextualizado histórica e geograficamente. O clima é resultante de interatividade e sujeito às mudanças que emergem de convivência organizacional.

O clima organizacional pode ser definido como o compartilhamento de percepções sobre as políticas, práticas, procedimentos e experiência dos empregados (OSTROFF et al. 2003, SCHNEIDER, EHRHART e MACEY, 2013).

Por outro lado, a cultura organizacional, de acordo com Schein (1992) pode ser definida como um padrão de suposições básicas demonstradas; inventadas, descobertas ou desenvolvidas por um dado grupo; que ensina a lidar com seus problemas externos de

ReLAInEP - Revista Latino-America de Inovação e Engenharia de Produção, Curitiba, PR, Brasil, v. 3, n. 4, p. 131-151, 2015. 


\section{ReLAInEP}

adaptação e internos de integração; que funcionou bem o bastante para ser considerado válido e, ainda, para ser ensinado aos novos membros do grupo como a forma correta de perceber, pensar e sentir em relação àqueles problemas. Para Hofstede (1997), a cultura organizacional pode ser entendida como a programação coletiva da mente, que distingue membros de uma organização de membros de outra.

Sendo assim, a cultura organizacional representa o sistema de comportamentos, normas e valores sociais aceitos e partilhados por todos os membros da organização e que de certa forma a tornam única (SMIRCICH, 1983; KOTTER \& HESKETT, 1992).

Os estudos sobre clima organizacional foram predominantes nas décadas de 1960 e 1970 na área de gestão de pessoas, sendo que nos anos 1980 e 1990 o foco das pesquisas foi a cultura organizacional. Recentemente, de acordo com Schneider, Ehrhart e Macey (2013) os estudos sobre clima organizacional têm voltado à tona nas discussões organizacionais.

Tem-se que o clima organizacional pode ser medido separadamente por um processo de escores individuais em um levantamento de clima e depois esses escores podem ser agregados para as unidades que compõem a organização como um todo, ou seja, o clima organizacional seria uma forma mais imediata da cultura se manifestar na organização (SANTOS, 2012).

\subsection{Variáveis que afetam o grau de satisfação e motivação dos trabalhadores}

$\mathrm{Na}$ perspectiva de analisar o clima organizacional, diversas variáveis foram identificadas na literatura. O clima organizacional depende de fatores internos e externos, tais como supervisão, salário, desenvolvimento profissional, feedback e trabalho em si entre outros, estes fatores induzem a motivação provocando níveis diferentes de satisfação, sendo revertido em termos de eficácia e eficiência dos funcionários da organização, resultando no serviço prestado aos clientes

- Supervisão

ReLAInEP - Revista Latino-America de Inovação e Engenharia de Produção, Curitiba, PR, Brasil, v. 3, n. 4, p. 131-151, 2015. 


\section{ReLAInEP}

A variável supervisão trata de gestão, processo decisório e liderança de gestão, sendo assim referenciada a satisfação dos funcionários com os seus gestores no que se refere à capacidade técnica e administrativa, as formas e canais de comunicação e o tratamento dispensado ao grupo de trabalho (LUZ, 2007). A liderança é essencial em todas as funções, prevê o alcance de objetivos e consiste em saber conduzir as pessoas ou grupos pela influência interpessoal e pelo processo de comunicação humana (CHIAVENATO, 2006).

Rahim (1989 apud RESENDE, MARTINS e SIQUEIRA 2010) define poder do supervisor como a habilidade para mudar ou controlar o comportamento, atitudes, opiniões, objetivos, necessidades e valores de seu subordinado. Para exercer este poder, o supervisor pode usar de fontes diferenciadas a fim de influenciar seus subordinados.

De acordo com Pedroso (2010), muitos autores fazem associação de liderança com o fato de influenciar pessoas a fim de realizar determinada meta. Para que essas metas sejam alcançadas, um líder tem de ser capaz de relacionar-se de forma eficaz com muitas pessoas, sejam elas superiores, colegas, clientes e subordinados, especialmente com esse último grupo (MEGGINSON, MOSLEY e PIETRY JR., 1998; BERGAMINI,1994; PEDROSO, 2010).

\section{- Salário}

Esta é uma das mais importantes variáveis a ser considerada em uma pesquisa de clima organizacional, corresponde a benefícios e a remuneração, haja vista sua forte influência no grau de satisfação dos funcionários. Neste estudo, esta variável foi abordada visando identificar a satisfação dos servidores quanto ao salário percebido, aos benefícios e ao Plano de Cargos e Vencimentos (LUZ, 2007).

O objetivo de um sistema de remuneração segundo Milkovic e Boudreau (2000) é motivar comportamentos produtivos e controlar os custos trabalhistas, ao mesmo tempo em que busca a satisfação das necessidades e do sentimento de justiça dos empregados.

"Salário é uma contraprestação por serviços prestados. Em troca de dinheiro a pessoa empenha parte do seu esforço e de sua vida, comprometendo-se a uma atividade e a um padrão de desempenho na organização" (CHIAVENATO, 2006, p. 224) "Benefícios são componentes que constituem uma forma indireta de remuneração, destinada a proporcionar melhor qualidade de vida aos funcionários" (SOUZA et al., 2005, p. 28).

ReLAInEP - Revista Latino-America de Inovação e Engenharia de Produção, Curitiba, PR, Brasil, v. 3, n. 4, p. 131-151, 2015. 


\section{ReLAInEP}

No setor público "a carreira, em geral, é definida como a ordenação sequencial de cargos especificada por níveis e classes" (BERGUE; 2005, p.218). Diversos são os aspectos relacionados à carreira dos servidores públicos, a saber:

Promoção - entendida como a evolução do servidor nos níveis e nas classes da carreira, processada por antiguidade ou merecimento, observando-se, neste caso, os critérios de qualificação e eficiência funcional;

Níveis e classes - são gradientes remuneratórios vinculados a um cargo, cuja progressão é, em geral, associada à aquisição de diferenciais no grau de escolaridade e habilitação por parte do servidor;

Eficiência funcional - trata-se de critério associado ao desempenho do agente público no exercício do cargo em que esteja investido, a ser evidenciado mediante processo de avaliação formal na forma de regulamento próprio (BERGUE, 2005, p. 222).

\section{- Desenvolvimento profissional}

Esta variável avalia as oportunidades e acesso dos funcionários a cursos de qualificação ou treinamento que visam contribuir com o desenvolvimento pessoal e organizacional (LUZ, 2007).

Chiavenato (2006) se refere ao desenvolvimento organizacional como uma mudança planejada da organização que parte de uma visão global e sistêmica e busca, com uma atuação construtiva junto aos processos e estrutura da organização, melhorar seu desempenho.

Já o desenvolvimento de pessoal nas organizações pode ser definido como "programas de longo prazo para prover o crescimento profissional das pessoas por meio de condições externas capazes de realizar, gradativamente, as potencialidades humanas" (CHIAVENATO, 2006, p. 182). Para Tachizawa, Ferreira e Fortuna (2004) as organizações identificam a educação como um processo essencial, tanto de complementação do ensino formal, quanto de disseminação de princípios, conhecimentos e desenvolvimento das competências necessárias ao conjunto da organização e à vida profissional.

\section{- Feedback}

ReLAInEP - Revista Latino-America de Inovação e Engenharia de Produção, Curitiba, PR, Brasil, v. 3, n. 4, p. 131-151, 2015. 


\section{ReLAInEP}

Esta variável destaca a estabilidade no emprego, o processo decisório, as condições físicas do trabalho que são fatores influentes indesejáveis em conflitos internos, de acordo com Luz (2007) apresenta esta variável como uma maneira de avaliar a qualidade das relações pessoais e a intensidade dos conflitos eventualmente existentes no ambiente de trabalho.

Todavia, de acordo com Chiavenato (2006) as pessoas nas organizações nem sempre tem objetivos e interesses comuns. Este fato acarreta divergências que podem gerar conflitos de três diferentes níveis, a saber:

- Conflito percebido - ocorre quando as partes percebem a existência do conflito porque há divergência dos objetivos. Chama-se latente, pois sua percepção é potencial;

- Conflito experimentado - sua ocorrência se manifesta por meio de sentimentos de hostilidade, raiva ou medo. É um conflito velado, pois sua demonstração é dissimulada, não é externado claramente;

- Conflito manifestado - neste caso o conflito é abertamente demonstrado pelo comportamento das pessoas. Para o mesmo autor, nas organizações existem certas condições, chamadas de antecedentes, que são causa dos conflitos. Estas condições foram apresentadas por ele da seguinte maneira:

- Ambiguidade de papel - ocorre quando não existe clareza no que se refere às expectativas o que pode provocar nas pessoas o sentimento de estarem trabalhando para propósitos incompatíveis;

- Objetivos concorrentes - ocorre quando a organização cresce e os grupos tendem a se especializar cada vez mais na tentativa de alcançar seus objetivos. Esses objetivos e interesses podem ser divergentes entre os grupos o que gera conflitos;

- Recursos compartilhados - quando os recursos disponíveis se tornam limitados e escassos e precisam ser distribuídos entre os grupos da organização. Para um grupo aumentar seus recursos o outro terá que perder alguma coisa, tornando objetivos e interesses incompatíveis;

- Interdependência de atividades - quando os grupos ou pessoas de uma organização dependem entre si para a realização de suas atividades. Normalmente existe entre os grupos algum nível de interdependência.

ReLAInEP - Revista Latino-America de Inovação e Engenharia de Produção, Curitiba, PR, Brasil, v. 3, n. 4, p. 131-151, 2015. 


\section{ReLAInEP}

- Trabalho em si.

Esta variável está relacionada à cultura organizacional, ao trabalho em equipe e a participação. Enquanto a cultura organizacional se mantém durante toda a existência de uma organização ou, pelo menos durante parte dela, apontado os caminhos a serem seguidos em determinadas etapas, o clima organizacional se modifica conjunturalmente conforme os diferentes momentos pelo quais passam as organizações (TACHIZAWA; FERREIRA; FORTUNA, 2004, p.241)

De acordo com Terra (2005, p.120), a "cultura organizacional pode ser entendida pelas normas e valores que ajudam a interpretar eventos e avaliar o que é apropriado e inapropriado".

Para Silva (2001, p.421) "a cultura organizacional é o sistema de valores, crenças, normas e hábitos compartilhados, que rege a interação dos elementos de uma organização".

Pode-se representar a cultura organizacional pela missão e valores da empresa. "A cultura organizacional deve ser ancorada e construída por meio de atos simbólicos e ações da alta administração que valorizam e destacam fatos, notícias e pessoas que exemplificam os valores perseguidos" (TERRA, 2005, p. 120). É importante que as organizações tenham consciência de que é preciso constantemente fomentar a missão e os valores das mesmas, bem como deixar claro como esses elementos se encaixam na estratégia adotada pela organização.

\section{- Estresse - Adaptação dos trabalhadores}

De acordo com Palmer, Cooper e Thomas (2003) o estresse ocorre quando a pressão excede a habilidade de enfrentar as dificuldades. Diversos componentes do clima podem afetar de forma significativa o estresse no trabalho, influenciando negativamente a saúde e o bem-estar do empregado.

Para Straub (2005) existem alguns fatores que tornam certos trabalhos mais estressantes do que outros:

a) sobrecarga de trabalho - pessoas que trabalham em muitas tarefas e geralmente apresentam os piores hábitos de saúde, experimentam mais acidentes e sofrem de mais problemas de saúde do que os outros. Mas é importante observar que o número de horas que uma pessoa trabalha não é indicador confiável de estresse. E a sobrecarga de trabalho apresenta um componente subjetivo além do componente objetivo. Um trabalhador pode

ReLAInEP - Revista Latino-America de Inovação e Engenharia de Produção, Curitiba, PR, Brasil, v. 3, n. 4, p. 131-151, 2015. 
sentir um grande peso no seu horário ou pela atividade que executa e outro pode ser capaz de lidar de forma fácil com a situação;

b) sobrecarga de papéis - ocorre quando as pessoas exercem vários papéis ao mesmo tempo, principalmente entre as mulheres. Straub cita um estudo de Williams, Suls, Alliger, Learner e Wan (1991) que explora a sobrecarga das mães trabalhadoras. Os resultados demonstraram sobrecarga de papéis, pelo fato de lidar com as tarefas do trabalho e do lar, redução do aproveitamento de todas as tarefas e piora do humor. Entretanto, outras pesquisas citadas por Straub demonstram que as mulheres trabalhadoras são geralmente mais saudáveis do que as desempregadas. O emprego é uma importante fonte de auto-estima e de satisfação na vida. Por isso, não é o número de papéis que uma mulher ocupa que vai causar estresse ou alteração de humor, mas a qualidade de sua experiência nesses papéis e o controle sobre o próprio trabalho;

c) esgotamento - estado de exaustão física e psicológica relacionada com o trabalho. É uma síndrome multidimensional caracterizada por exaustão emocional, despersonalização e redução em realizações pessoais. O esgotamento é mais comum em empregos que envolvem responsabilidade com outras pessoas, como, por exemplo, os trabalhadores da área de saúde; d) falta de controle sobre o trabalho - ocorre mais estresse quando os trabalhadores têm pouco ou nenhum controle sobre os procedimentos, o ritmo e outros aspectos de seu trabalho. As Secretárias, garçonetes, operários e gerentes intermediários estão entre as ocupações mais estressantes, visto que as tarefas são repetitivas e que há pouco controle sobre os eventos;

e) outras fontes de estresse relacionado com o trabalho - outros aspectos verificados que aumentam o estresse entre os trabalhadores - Ambiguidade ou conflito de papéis (não tem certeza do seu trabalho ou dos padrões utilizados para avaliar o seu desempenho), turnos de revezamento, perda do emprego, assédio sexual, assédio moral etc.

Outro fator importante relacionado ao estresse organizacional é o impacto das políticas organizacionais. A percepção de um clima politicamente negativo. As respostas dos trabalhadores aos estressores laborais e organizacionais são do tipo fisiológico, psicológico e comportamental. Contudo, os estudos científicos mostram que existem diferenças individuais importantes na reação dos trabalhadores às exigências do trabalho. Ou seja, trabalhadores expostos aos mesmos estressores laborais e organizacionais apresentam fatores disposicionais

ReLAInEP - Revista Latino-America de Inovação e Engenharia de Produção, Curitiba, PR, Brasil, v. 3, n. 4, p. 131-151, 2015. 


\section{ReLAInEP}

diferentes (variáveis afetivas como a disposição e a afetividade negativa) e maneira de enfrentar as situações estressantes também diferentes (STRAUB, 2005).

A seguir será detalhada a metodologia do trabalho, com destaque para informações sobre o instrumento de coleta de dados e as análises que foram realizadas.

\section{METODOLOGIA}

Este estudo foi realizado em três organizações de ramos distintos localizadas no estado do Paraná. A empresa A fornece serviços e tecnologias para uma instituição bancária, a empresa B é responsável por manter serviços essenciais em um município como limpeza pública e a empresa $\mathrm{C}$ é uma transportadora de grãos.

Trata-se de uma pesquisa exploratória com ênfase quantitativa, cuja amostra consistiu apenas de trabalhadores do setor operacional das três empresas, sendo que na A correspondeu a 81 trabalhadores, na empresa B 104 trabalhadores e a empresa C 38 pessoas.

Neste estudo, o instrumento de pesquisa foi baseado no modelo de Luz (1995), considerando as variáveis: a supervisão, o salário, o desenvolvimento profissional, o feedback, o trabalho em si e estresse. Os quesitos benefícios e estabilidade no emprego foram tratados nas variáveis salário e supervisão, respectivamente.

Os dados primários foram tratados utilizando-se uma estatística descritiva e análise de médias simples. Para a realização da análise descritiva, os dados obtidos por meio das vinte e duas questões, foram agrupados numa escala tipo Likert de dez pontos, conforme seu grau de importância. As dimensões de importância foram classificadas em: 1 a 2 péssimo, de 3 a 4 ruim, de 5 a 6 regular, de 7 a 8 bom, e de 9 a 10 ótimo

O instrumento de coleta dos dados foi o questionário, contendo 22 questões objetivas, abrangendo as seguintes variáveis: avaliação da supervisão de 01 a 08, avaliação do salário de 9 a 10, avaliação do desenvolvimento profissional de 11 a 14, avaliação do feedback de 15 a 20, avaliação do trabalho em si de 21 a 22.

Os dados foram analisados por meio da estatística descritiva utilizando a média, o desvio padrão e do coeficiente de variação. O coeficiente de variação mede a dispersão em termos de percentagem e não em unidades de medida. Assim, esta relação foi utilizada para comparar a dispersão entre as três empresas. Coeficientes menores que $10 \%$ foram

ReLAInEP - Revista Latino-America de Inovação e Engenharia de Produção, Curitiba, PR, Brasil, v. 3, n. 4, p. 131-151, 2015. 


\section{ReLAInEP}

considerados baixos, indicando uma homogeneidade nas respostas, de $10 \%$ a $20 \%$ foram considerados médios e acima de $30 \%$ foram considerados altos, indicado uma elevada heterogeneidade nas três empresas analisadas.

A variável estresse foi mensurada por meio da frequência de ocorrência - Sempre; quase sempre; às vezes; Raramente e Nunca -, sendo que estes valores foram transformados em uma escala de 10 a 0 , multiplicando os valores percentuais respectivamente por $10 ; 7,5 ; 5$; 2,5 e 0.

\section{RESULTADOS ENCONTRADOS}

A Tabela 1 apresenta as questões que analisam os superiores imediatos nas três empresas estudadas. Tem-se que a Empresa A apresentou média geral de 8,37 para estas questões e a empresa B de 7,50 sendo estes valores classificados como Bons. A empresa C apresentou média 5,4 considerada regular. O coeficiente de variação entre as três empresas foi de $20 \%$, considerado médio, sendo que a divergência de resultados encontrados era esperada devido ao fato da pesquisa ter sido realizada com empresas de ramo de atividades diferentes. Prosseguindo com a comparação entre as três empresas, podem-se observar algumas semelhanças.

Tabela 1 - Questões que analisam os superiores imediatos nas empresas selecionadas

\begin{tabular}{|c|c|c|c|c|c|c|}
\hline Questões / Empresas & $\mathbf{A}$ & $\mathbf{B}$ & $\mathbf{C}$ & Média & $\begin{array}{l}\text { Desvio } \\
\text { Padrão }\end{array}$ & $\begin{array}{c}\text { Coeficiente } \\
\text { de } \\
\text { Variação } \\
\end{array}$ \\
\hline $\begin{array}{l}\text { Seu superior imediato costuma atender as suas } \\
\text { reclamações? }\end{array}$ & 8,11 & 7,10 & 5,13 & 6,78 & 1,42 & 0,21 \\
\hline $\begin{array}{l}\text { Seu superior imediato tem lhe dado orientação de forma } \\
\text { adequada? }\end{array}$ & 8,15 & 7,57 & 5,62 & 7,11 & 1,39 & 0,20 \\
\hline Seu superior imediato trata você com o devido respeito? & 8,98 & 7,96 & 6,46 & 7,80 & 1,46 & 0,19 \\
\hline Questões / Empresas & $\mathbf{A}$ & B & $\mathbf{C}$ & Média & $\begin{array}{l}\text { Desvio } \\
\text { Padrão }\end{array}$ & $\begin{array}{c}\text { Coeficiente } \\
\text { de } \\
\text { Variação }\end{array}$ \\
\hline $\begin{array}{l}\text { Você acha que seu superior imediato conhece os objetivos } \\
\text { e metas do seu setor? }\end{array}$ & 8,44 & 7,88 & 5,45 & 7,25 & 1,31 & 0,18 \\
\hline $\begin{array}{l}\text { Como está o seu relacionamento com o seu superior } \\
\text { imediato? }\end{array}$ & 8,69 & 7,78 & 6,41 & 7,63 & 1,28 & 0,17 \\
\hline $\begin{array}{l}\text { Seu superior informa como você está indo no desempenho } \\
\text { do seu trabalho? }\end{array}$ & 8,21 & 7,03 & 4,73 & 6,66 & 1,36 & 0,20 \\
\hline Seu superior procura fazer seu setor trabalhar unido? & 8,05 & 7,89 & 4,95 & 6,96 & 1,83 & 0,26 \\
\hline Seu superior imediato aceita sugestões para a melhoria do & 8,31 & 6,83 & 4,45 & 6,53 & 1,45 & 0,22 \\
\hline
\end{tabular}

ReLAInEP - Revista Latino-America de Inovação e Engenharia de Produção, Curitiba, PR, Brasil, v. 3, n. 4, p. 131-151, 2015. 
trabalho?

Média Geral para a variável Supervisão

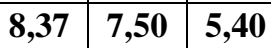

7,09

Fonte: Dados coletados pelos autores

$\mathrm{Na}$ sequência, foram analisados os itens que apresentaram um nível de satisfação acima da média geral de cada empresa. Obteve-se similaridade positiva de respostas nas três empresas, nos seguintes itens: $\mathrm{O}$ superior imediato trata o encarregado com devido respeito; o superior imediato conhece os objetivos e metas do seu departamento (setor); e é satisfatório o relacionamento entre superior imediato e encarregado. No segundo momento, foi identificado o nível de satisfação em relação a supervisão que está abaixo da média geral de cada empresa, onde obteve-se similaridade negativa nas respostas entre as três empresas, sendo os seguintes itens: $\mathrm{O}$ superior imediato não aceita sugestões para a melhoria do trabalho; o superior imediato não informa o desempenho dos funcionários; e superior imediato não atende as reclamações dos funcionários.

Em relação às questões que analisam o salário, todas as três (3) empresas obtiveram valores médios considerados regular, conforme Tabela 2. O coeficiente de variação nas três empresas foi de 32\%, considerado alto. Cabe destacar que a empresa $\mathrm{C}$ obteve valor 3,24, considerado ruim, para a questão que mensurou o atendimento das necessidades básicas dos colaboradores.

Tabela 2 - Questões que analisam os salários nas empresas selecionadas

\begin{tabular}{c|c|c|c|c|c|c}
\hline Questões / empresas & A & B & C & Média & $\begin{array}{c}\text { Desvio } \\
\text { Padrão }\end{array}$ & $\begin{array}{c}\text { Coeficiente } \\
\text { de Variação }\end{array}$ \\
\hline $\begin{array}{c}\text { O seu salário é suficiente para atender suas necessidades } \\
\text { básicas? }\end{array}$ & 5,27 & 6,46 & 3,24 & 4,99 & 1,64 & 0,33 \\
\hline $\begin{array}{c}\text { Você está satisfeito com o plano de saúde que a empresa } \\
\text { lhe oferece? }\end{array}$ & 5,74 & 5,14 & 4,89 & 5,26 & 1,65 & 0,31 \\
\hline Salario & $\mathbf{5 , 5 1}$ & $\mathbf{5 , 8}$ & $\mathbf{4 , 0 6}$ & $\mathbf{5 , 1 2}$ & $\mathbf{1 , 6 4}$ & $\mathbf{0 , 3 2}$ \\
\hline
\end{tabular}

Fonte: Dados coletados pelos autores

A análise das questões que compõem o "Desenvolvimento Profissional", informadas na Tabela 3, classifica a Empresa A como bom e as Empresas B e C com resultado regular, o coeficiente de variação médio entre as três empresas foi de $31 \%$, considerado alto.

Tabela 3 - Questões que analisam o Desenvolvimento Profissional nas empresas selecionadas

\begin{tabular}{c|c|c|c|c|c|c}
\hline Questões / empresas & A & B & C & Média & $\begin{array}{c}\text { Desvio } \\
\text { Padrão }\end{array}$ & $\begin{array}{c}\text { Coeficiente } \\
\text { de Variação }\end{array}$ \\
\hline $\begin{array}{c}\text { Os materiais (maquinas/equipamentos) que você usa para } \\
\text { executar o seu trabalho favorece o seu desenvolvimento }\end{array}$ & 7,74 & 6,87 & 6,42 & 7,01 & 1,51 & 0,22 \\
\hline
\end{tabular}

ReLAInEP - Revista Latino-America de Inovação e Engenharia de Produção, Curitiba, PR, Brasil, v. 3, n. 4, p. 131-151, 2015. 


\begin{tabular}{c|c|c|c|c|c|c}
\hline \multicolumn{1}{|c}{ profissional? } & & & & & \\
\hline $\begin{array}{c}\text { Ps. } \\
\text { As promoções da empresa são dadas a quem merece? }\end{array}$ & 5,18 & 5,44 & 4,23 & 4,95 & 1,9 & 0,38 \\
\hline $\begin{array}{c}\text { A empresa tem lhe dado oportunidade de } \\
\text { desenvolvimento profissional (treinamentos)? }\end{array}$ & 5,93 & 5,3 & 2,89 & 4,71 & 1,66 & 0,35 \\
\hline $\begin{array}{c}\text { Os treinamentos que você recebe tem melhorado na ajuda } \\
\text { de seu trabalho? }\end{array}$ & 6,17 & 5,7 & 4,47 & 5,45 & 1,77 & 0,33 \\
\hline Desenvolvimento profissional & $\mathbf{6 , 2 5}$ & $\mathbf{5 , 8 3}$ & $\mathbf{4 , 5}$ & $\mathbf{5 , 5 3}$ & $\mathbf{1 , 7 1}$ & $\mathbf{0 , 3 1}$ \\
\hline
\end{tabular}

Fonte: Dados coletados pelos autores

Analisando-se os itens que apresentam nível de satisfação acima da média geral de cada empresa, obteve-se similaridade positiva de respostas entre as três empresas, apenas em um item: os materiais (maquinas/ equipamentos) fornecidos pela empresa que são utilizados para execução do trabalho favorece ao bom desenvolvimento profissional. As questões que mais contribuíram para apresentar valores baixos nos resultados encontrados referem-se ao merecimento das promoções realizadas pela empresa e aos treinamentos disponibilizados pelas mesmas.

Os itens analisados em Feedback categorizam as Empresas A e B como bom e a Empresa C como regular, conforme Tabela 4. O coeficiente de variação entre as três empresas foi de $24 \%$, considerado médio. Analisando todos os itens que apresentam nível de satisfação acima da média geral de cada empresa, obteve-se similaridade positiva de respostas entre as três empresas, apenas em um item: quanto a satisfação o entrevistado está em relação a vida pessoal (amigos, família, social). O nível de satisfação em relação ao desenvolvimento profissional que está abaixo da média geral nas três empresas analisadas são: motivações oferecidas pela empresa (cursos, palestra, promoções); e a satisfação referente ao apoio recebido no trabalho.

Tabela 4 - Questões que analisam o Feedback nas empresas selecionadas

\begin{tabular}{c|c|c|c|c|c|c}
\hline Questões / empresas & A & B & C & Média & $\begin{array}{c}\text { Desvio } \\
\text { Padrão }\end{array}$ & $\begin{array}{c}\text { Coeficiente } \\
\text { de } \\
\text { Variação }\end{array}$ \\
\hline $\begin{array}{c}\text { Você está satisfeito com o seu ambiente de trabalho? } \\
\text { (iluminação/ventilação) }\end{array}$ & 6,9 & 7,59 & 6,06 & 6,85 & 1,51 & 0,22 \\
\hline
\end{tabular}

ReLAInEP - Revista Latino-America de Inovação e Engenharia de Produção, Curitiba, PR, Brasil, v. 3, n. 4, p. 131-151, 2015. 


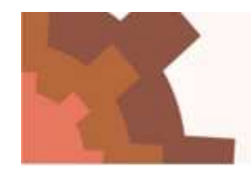

ReLAInEP

\begin{tabular}{|c|c|c|c|c|c|c|}
\hline $\begin{array}{l}\text { Qual sua avaliação das motivações oferecidas pela } \\
\text { empresa de (cursos, palestras)? }\end{array}$ & 5,62 & 5,87 & 3,18 & 4,89 & 1,71 & 0,35 \\
\hline Você se sente satisfeito em trabalhar na empresa? & 6,91 & 8,47 & 4,84 & 6,74 & 1,74 & 0,26 \\
\hline Quanto você está satisfeito com sua vida pessoal? & 8,62 & 8,73 & 8,14 & 8,49 & 1,34 & 0,16 \\
\hline $\begin{array}{l}\text { Quanto satisfeito você está com o apoio que recebe do } \\
\text { trabalho? }\end{array}$ & 6,95 & 7,36 & 4,51 & 6,27 & 1,65 & 0,26 \\
\hline $\begin{array}{l}\text { Qual a sua opinião sobre a sua higiene e organização do } \\
\text { ambiente de trabalho? }\end{array}$ & 7,93 & 6,53 & 6,81 & 7,09 & 1,63 & 0,23 \\
\hline Feedback & 7,15 & 7,43 & 5,59 & 6,72 & 1,59 & 0,24 \\
\hline
\end{tabular}

Fonte: Dados coletados pelos autores

Em relação ao trabalho em si, constatou-se que a empresa $\mathrm{B}$ é considerado como Bom, ao passo que nas empresas este item é considerado Regular e na empresa C é considerado Ruim. O coeficiente de variação foi bem elevado, de $55 \%$, demonstrando a heterogeneidade nas respostas entre os colaboradores das três empresas analisadas.

Tabela 5 - Questões que analisam o Trabalho em Si nas empresas selecionadas

\begin{tabular}{c|c|c|c|c|c|c}
\hline Questões / empresas & A & B & C & Média & $\begin{array}{c}\text { Desvio } \\
\text { Padrão }\end{array}$ & $\begin{array}{c}\text { Coeficiente } \\
\text { de } \\
\text { Variação }\end{array}$ \\
\hline $\begin{array}{c}\text { Você aconselharia algum amigo seu a trabalhar na } \\
\text { empresa? }\end{array}$ & 6,88 & 8,15 & 4,83 & 6,62 & 3,63 & 0,55 \\
\hline
\end{tabular}

Fonte: Dados coletados pelos autores

O estresse foi mensurado nas três empresas selecionadas. Os valores da Tabela 6 referem-se à frequência da ocorrência de sentimentos negativos em relação ao trabalho. Os valores foram transformados em um indicador de 0 a 10 para facilitar a compreensão.

Tabela 6 - Avaliação do Estresse nas empresas selecionadas (\%)

\begin{tabular}{l|c|c|c|c|c|c}
\hline $\begin{array}{l}\text { Você tem sempre sentimentos } \\
\text { negativos tais como: mau humor, } \\
\text { desespero, ansiedade, depressão } \\
\text { relacionados ao trabalho? }\end{array}$ & \multicolumn{7}{c|}{\begin{tabular}{c} 
\\
Pempr \\
\cline { 2 - 8 }
\end{tabular}} & $\begin{array}{c}\text { Quase } \\
\text { sempre }\end{array}$ & As vezes & Raramente & Nunca & Indicador \\
\hline Empresa A & 2 & 6 & 31 & 45 & 16 & 3,33 \\
\hline Empresa B & 2 & 9 & 37 & 29 & 24 & 3,45 \\
\hline Empresa C & 18 & 16 & 39 & 11 & 16 & 5,23 \\
\hline Percentual Total (\%) & 7 & 10 & 36 & 28 & 19 & 4,00 \\
\hline
\end{tabular}

ReLAInEP - Revista Latino-America de Inovação e Engenharia de Produção, Curitiba, PR, Brasil, v. 3, n. 4, p. 131-151, 2015. 


\section{ReLAInEP}

A Empresa $\mathrm{C}$ apresenta o maior nível de estresse em relação às demais organizações. Os valores de 3,33 encontrado na Empresa A e 3,45 na Empresa B indicam que raramente os sentimentos negativos ocorrem nestas duas organizações.

A fim de resumir os resultados encontrados, a Tabela 7 e o Gráfico 1 apresentam os valores encontrados nas variáveis supervisão, salário, desenvolvimento profissional, feedback e trabalho em si coletados para as três empresas.

Tabela 7 - Resumo das variáveis analisadas (\%)

\begin{tabular}{l|l|l|l}
\hline \multicolumn{1}{c|}{ Variáveis } & \multicolumn{1}{c|}{ Empresa A } & \multicolumn{1}{c|}{ Empresa B } & \multicolumn{1}{c}{ Empresa C } \\
\hline Supervisão & 8,37 & 7,5 & 5,4 \\
\hline Salário & 5,51 & 5,8 & 4,06 \\
\hline Desenvolvimento profissional & 6,25 & 5,83 & 4,5 \\
\hline Feedback & 7,15 & 7,43 & 5,59 \\
\hline Trabalho em si & 6,88 & 8,15 & 4,83 \\
\hline Média Geral & 6,83 & 6,94 & 4,88 \\
\hline
\end{tabular}

Fonte: Dados coletados pelos autores

A média geral das cinco variáveis analisadas indicam que o clima organizacional nas Empresas B e A são considerados regulares, com valores próximos ao indicador 7, considerado bom e na Empresa $\mathrm{C}$ é considerado ruim, sendo que as variáveis salários e desenvolvimento profissional foram as que mais contribuíram negativamente para a avaliação do clima e a variável supervisão foi a que mais influenciou positivamente. Esses resultados corroboram os valores de estresse informados na Tabela 7, ou seja, há evidência de que maiores níveis de estresse geram um nível baixo de satisfação e consequente clima organizacional.

Gráfico 1 - Comparativo dos valores encontrados nas 5 variáveis analisadas para as empresas A, B e C

ReLAInEP - Revista Latino-America de Inovação e Engenharia de Produção, Curitiba, PR, Brasil, v. 3, n. 4, p. 131-151, 2015. 


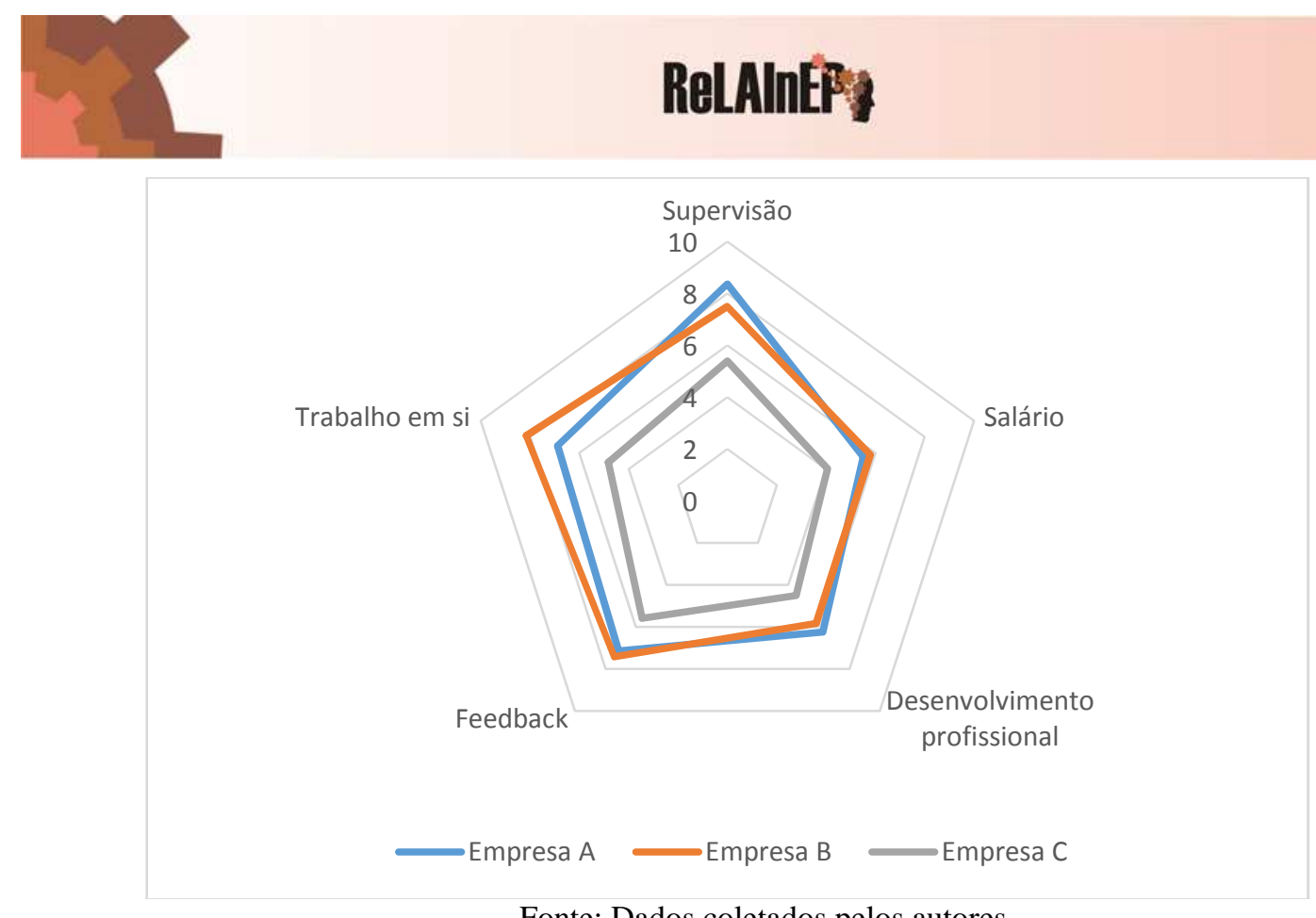

Fonte: Dados coletados pelos autores

\section{CONSIDERAÇÕES FINAIS}

Este artigo procurou analisar o clima organizacional de três empresas de ramo e atividades distintas na percepção dos colaboradores que atuam em setores operacionais.

Entender como as pessoas percebem o ambiente no qual trabalham contribui para que os gestores possam lidar melhor com os problemas cotidianos das organizações, com as questões motivacionais canalizando os esforços para que os objetivos sejam atingidos.

Os resultados obtidos possibilitaram algumas inferências gerais sobre os ambientes analisados. O clima organizacional é resultado de interpretações individuais e, portanto, singular e imerso de subjetividades. Cada colaborador traz consigo suas crenças, valores e modelos que podem interferir ou somar-se ao ambiente organizacional, facilitando ou dificultando as relações de trabalho. Os gestores são peças fundamentais para que as variáveis: supervisão, salário, desenvolvimento profissional, feedback e trabalho em si que afetam o clima organizacional façam parte das políticas de gestão de pessoas, favorecendo a concretização dos objetivos e das ações.

As empresas estudadas embora de ramo e atividades distintas apresentaram, em algumas variáveis, perfis muito similares tanto do ponto de vista positivo quanto negativo. Cabe destacar que o estresse está relacionado com os valores encontrados para o clima organizacional nas três empresas analisadas.

ReLAInEP - Revista Latino-America de Inovação e Engenharia de Produção, Curitiba, PR, Brasil, v. 3, n. 4, p. 131-151, 2015. 


\section{ReLAInEP}

Sugere-se que outros estudos sejam realizados em empresas de ramo e ou atividades similares, buscando-se compreender como o clima organizacional pode afetar o desempenho. Outra questão a ser estudada é a análise de ambientes de empresas consideradas inovadoras e criativas, utilizando-se, por exemplo, a escala de Kolb (1995).

\section{REFERÊNCIAS}

ABBEY, A.; DICKSON, J.W.R work climate and innovation in semiconductors. Academy of Management Journal, v.26, n.2, p.362-368, 1983.

ARAUJO, L. C. G.; GARCIA, A. A. Gestão de pessoas: estratégia e integração organizacional. 2ed. São Paulo: Atlas, 2009.

ASKANASY, N. P.; WILDEROM, C. P. M.; PETERSON, M. F. Handbook of organizational culture and climate. California: Sage Pub, 2000.

BEGNAMI, M.L.V; ZORZO, A. Clima organizacional: percepções e aplicabilidade. Revista Científica da FHO|UNIARARAS, v.1, n.2, 2013

BERGAMINI, Cecília W. Liderança: administração do sentido. 1. ed. São Paulo: Atlas, 1994.

BERGUE, Sandro Trescastro. Gestão de pessoas em organizações públicas. Caxias do Sul: Educs, 2005.

BRUXEL, A.; JUNQUEIRA, A.G.W. Gestão de pessoas: satisfação dos profissionais de uma organização do ramo de telecomunicações. Revista Destaques Acadêmicos, v. 3, n. 1, 2011.

CHIAVENATO, I. Gestão de pessoas: o novo papel dos recursos humanos nas organizações. Rio de Janeiro: Campus, 1999 Administração geral e pública. Rio de Janeiro: Elsevier, 2006.

DENISON D.R. What is the difference between organizational culture and organizational climate? A native's point of view on a decade of paradigm wars. Acad Manag Ver 1996;21(3):619-54

FOREHAND, G. A., GILMER, H. B. Environmental variation in studies of organizational behavior. Psychological Bulletin, v. 62, n.6, p. 361-382, 1964

GOMES MENEZES, I.; PASSOS GOMES, A. C. Clima organizacional: uma revisão histórica do construto. Psicol. rev. (Belo Horizonte), Belo Horizonte, v. 16, n. 1, abr. 2010. Disponível em $<$ http://pepsic.bvsalud.org/scielo.php?script=sci_arttext\&pid=S1677-

11682010000100011\&lng=pt\&nrm=iso >. acessos em 28 dez. 2014.

HELLRIEGEL D, SLOCUM JW. Organizational climate: measures, research and contingencies. Acad Manage, v.17, n.2, p.255-80, 1974.

HOFSTEDE, G. Cultures and Organizations: Software of the Mind. New York: McGraw-Hill, 1997.

KATZ, D.; KAHN, R. Psicologia Social das Organizações. 2. ed. São Paulo: Atlas, 1975.

KOLB, D. A. Organization behavior reader. New Jersey: Prentice Hall, 1995.

KOLB, D.; RUBIN, I. M; MCINTYRE, J. M. Psicologia Organizacional: uma abordagem vivencial. São Paulo: Atlas, 1978.

KOTTER J. P: HESKETT J. L. Corporate Culture and Performance. New York: Free Press, 1992.

ReLAInEP - Revista Latino-America de Inovação e Engenharia de Produção, Curitiba, PR, Brasil, v. 3, n. 4, p. 131-151, 2015. 


\section{ReLAInEP}

LEMOS, D.M.R; MARTINS, P.O. Avaliação de clima organizacional e a análise da relação entre a variável comportamento da chefia nas demais variáveis de clima: um estudo de caso em uma instituição financeira capixaba. In: ENANPAD, 31, 2007, Rio de Janeiro - RJ. Anais eletrônicos.

LEWIN. K; LIPPITT, R.; WHITE, R. K. Patterns of agressive behavior in experimentally created social climates. Journal of Social Psycology, 1939

LUZ, R. S. Clima organizacional. Rio de Janeiro: Qualitymark, 1995.

LUZ, R. Gestão do clima organizacional. Rio de Janeiro: Qualitymark, 2007.

MALHOTRA, Naresh K. Pesquisa de Marketing: uma orientação aplicada. $3^{\circ}$ ed. Porto Alegre: Bookman, 2001.

MEGGINSON, L C; MOSLEY, D C; PIETRI JR, P H. Administração: conceitos e aplicações. 4. ed. São Paulo: Harbra, 1998.

MILKOVICK, G. T.; BOUDREAU, J. Administração de Recursos Humanos. São Paulo: Atlas, 2000

OSTROFF C, KINICKI AJ, TAMKINS MM. Organizational culture and climate. In: BORMAN, W.C.; ILGEN, D. R: KLIMOSKI, R. J. Handbook of Psychology: Industrial and Organizational Psychology. New York: Wiley, 2003, v. 12, p. 565-93.

PALMER, S., COOPER, C.; THOMAS, K. Creating a Balance: Managing Stress. London: British Library, 2003.

PEDROSO, L. G. A influência do estilo de liderança no clima organizacional: um estudo de caso em uma instituição financeira. Trabalho de Conclusão de Curso (Graduação em Administração) Escola de Administração, Universidade Federal do Rio Grande do Sul, Porto Alegre, 2010.

RAHIM, M. A. Relationships of leader power to compliance and satisfaction with supervision, Evidence from a national sample of managers. Journal of Management, v.15, p.545-557, 1989.

RESENDE, P. C; MARTINS, M. C. F.; SIQUEIRA, M. M. M. Bem-estar no trabalho: influência das bases de poder do supervisor e dos tipos de conflito. Mudanças - Psicologia da Saúde, v.18 n.1-2, Jan-Dez, p. 47-57, 2010.

SANTOS, J. N. Clima organizacional na administração pública: análise do conceito nos termos de referência dos editais de licitação no Brasil para o fortalecimento do processo de gestão. In: CONGRESO INTERNACIONAL DEL CLAD SOBRE LA REFORMA DEL ESTADO Y DE LA ADMINISTRACIÓN PÚBLICA, 27. Cartagena, Colombia, 30 oct. - 2 Nov. 2012 Documento Libre.

SCHEIN, E. H. Organizational Culture and Leadership. 2. ed. San Francisco: Jossey-Bass, 1992.

SCHENEIDER, b; EHRHART, M. G., MACEY, W.H. Organizational Climate and Culture. Annu. Rev. Psychol. V. 64, p. 361-88, 2013

SILVA, R. O. Teorias da Administração - São Paulo: Pioneira Thomson Learning, 2001.

SMIRCICH L. Concepts of culture and organizational analysis. Adm. Sci. Q.,v. 28, p.339-58, 1983.

SOUZA, M. Z. A.et al. Cargos, carreiras e remuneração. Rio de Janeiro: Fgv, 2005.

STRAUB, R. Introdução à Psicologia da saúde. In: Psicologia da saúde. Porto Alegre: Artmed, 2005. Cap. 1.

TACHIZAWA, T.; FERREIRA, V.C. P.; FORTUNA, A. A. M. Gestão com pessoas: uma abordagem aplicada às estratégias de negócios. Rio de Janeiro: FGV, 2004.

ReLAInEP - Revista Latino-America de Inovação e Engenharia de Produção, Curitiba, PR, Brasil, v. 3, n. 4, p. 131-151, 2015. 


\section{ReLAInEP}

TERRA, J. C. C. Gestão do Conhecimento: o grande desafio empresarial. Rio de Janeiro: Elsevier, 2005.

ANALISYS OF ORGANIZATIONAL CLIMATE IN PARANÁ (BRAZIL):

A MULTIPLE CASE STUDY

ANÁLISIS DEL CLIMA ORGANIZACIONAL: UN ESTUDIO DE CASO MÚLTIPLE EN EMPRESAS DEL ESTADO DE PARANÁ

ReLAInEP - Revista Latino-America de Inovação e Engenharia de Produção, Curitiba, PR, Brasil, v. 3, n. 4, p. 131-151, 2015. 
ReLAInEP - Revista Latino-America de Inovação e Engenharia de Produção, Curitiba, PR, Brasil, v. 3, n. 4, p. 131-151, 2015. 